\title{
R Tool Analysis of Gripper Motor Rehabilitation for Post Stroke Therapy
}

\author{
A. Kalaiarasi ${ }^{\text {a }}$, L. Ashok Kumar ${ }^{\text {b }}$ \\ ${ }^{a}$ Department of Electrical and Electronics Engineering, Sri Shakthi Institute of Engineering and \\ Technology, Coimbatore-641062, Tamil Nadu, India. \\ ${ }^{a}$ Department of Electrical and Electronics Engineering, PSG College of Technology, Coimbatore- \\ 641004, Tamil Nadu, India. \\ * Corresponding Author: akalaiarasi@siet.ac.in
}

Received: 30-(09-2021, Revised: 11-10-2021, Accepted: 13-10-2021, Published: 30-10-2021

\begin{abstract}
Today, cerebral stroke issues are one of the most terrifying disorders in the clinical era, in which the most common people are highly affected. Worldwide, more than 20,00,000 individuals are exposed to stroke problems like hemiplegia, consistently, where $70 \%$ of them pass away at the instance of stroke. Among the survival group after the treatment, more than $85 \%$ of them are exposed to long term permanent disability. The affected community practice themselves to survive along with disability due to financial instability and reachability. While comparing with western standards, developing nations like India need keen attention to improve the medical standards. In order to treat the affected at the instance of stroke, home based methodologies shall be introduced for better performance and to improve the standard of daily living. This project involves a gripper motor based regulator for wrist and fingers incorporated with Arduino 328P. This device is a textile fabric tailored with 5 gripper motors to actuate each fingers as a part of post stroke rehabilitation.
\end{abstract}

Keywords: Gripper Motor, Rehabilitation, Post Stroke Therapy

Introduction and Epidemiology

In general, stroke disorders are classified into Ischemic and Haemorrhagic. The impact of stroke is dependent on the location of injury and its severity [1]. According to World Health Organization (WHO), the global statistics of stroke accounts to 60 to $90 \%$ of disability $[2,6]$. With reference to the developing nations have higher strike rate of stroke and the prevalence rate in international sides are 500-900/100000 and in India it is 100-300/100000[3]. The number of death rates are 6-7 Million globally and 1.3 million in India. The DALY (Disability Adjusted Life Year) rates are 30000000 globally and 6000000 in India [4]. Over the recent decades, 
standards of stroke cases are decremented by $2 \%$ in global map whereas there is an incremental rate of $6 \%$ in developing countries [5]. The global and Indian Scenario is listed in the Table 1.

Table 1 International and Indian Scenario of Incidence and Prevalence of Stroke

\begin{tabular}{|c|l|l|l|l|l|l|}
\hline & \multicolumn{3}{|c|}{ International Scenario } & \multicolumn{3}{c|}{ Indian Scenario } \\
\hline S. No. & Country & Incidental & Prevalence & State & Incidental & Prevalence \\
\hline 1 & America & 0.9 Million & 4.8 Million & Mumbai & 0.2 Million & 1.4 Million \\
\hline 2 & Africa & 0.7 Million & 1.6 Million & Kolkata & 0.1 Million & 1.2 Million \\
\hline 3 & South East Asia & 1.8 Million & 4.5 Million & West Bengal & 1.1 Million & 1.5 Million \\
\hline 4 & Western Pacific & 3.3 Million & 9.1 Million & Tamil Nadu & 1.3 Million & 1.6 Million \\
\hline
\end{tabular}

\section{Design Methodology}

The device integrates a reflexive exercise machine with kinetic exercise. The general block diagram is shown in Figure 1. The rehabilitation device consists of a battery, Arduino microcontroller, servo gripper motors, nylon threads and a textile based glove. The battery used to power the whole system is lead acid type. The control is programmed in high performance lower power Arduino Uno microcontroller chip, ATMEGA 328. Five servo gripper motors that rotate to up to 180 degree is used to actuate the fingers. The motor is connected to the fingers using strong nylon threads tailored to the cotton fabric glove. The force is transmitted through the strings to cause the flexion and extension of the fingers. The microcontroller is programmed to operate the motors in both individual and combination modes to attain different functionalities The functioning of this system shown in Figure 1 is involved in the actuation of the fingers responding to extension and flexion of the hand movement. The movement of the fingers is based on the signal received from the ports of Embedded code which drives the circuit and the control circuit is shown in figure 1 . The servo-gripper motor acts as an actuator which facilitates with self-lock mechanism to set with position, speed and force. The switch, when turned on, enables the servo-gripper motor to facilitate each finger with on and off control i.e flexion and extension, providing the essential torque at different angles even to hold a small object.

When the motors are rotated at specific speed at one direction, a linear pulling force is transmitted through the strings to cause the flexion of fingers and when they are rotated in the opposite direction, it causes release of the threads and thereby making the fingers to extend. The threads are selected to be of desired length and strength and are mounted on a textile based glove so as to provide support and comfort to the patient. This on and off control is extended to all the fingers thereby enabling the patient to hold and release an object in grip. 
A. Kalaiarasi et al., /2021

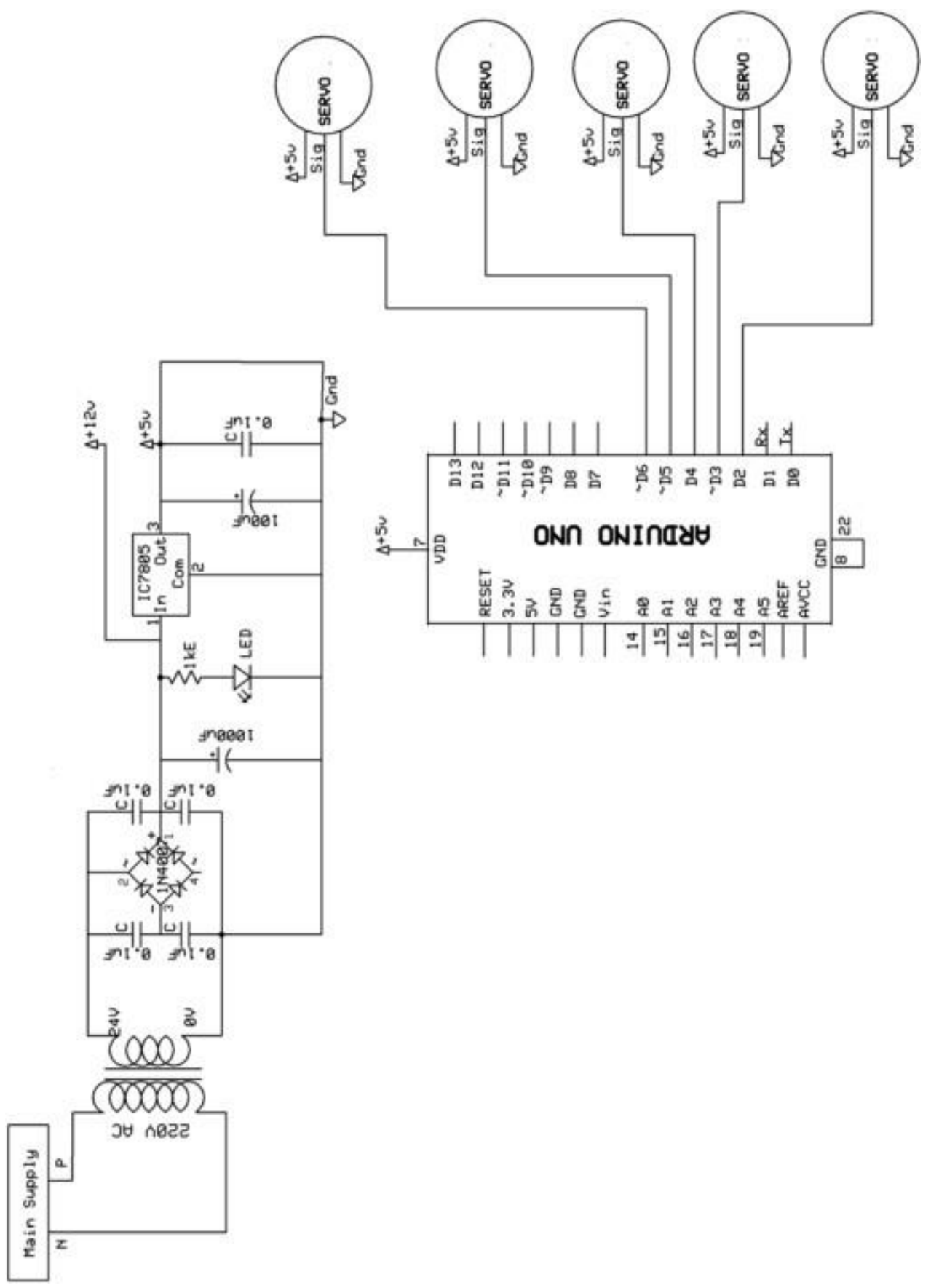

Figure 1 Control circuit of the Gripper motor for motor based rehabilitation 


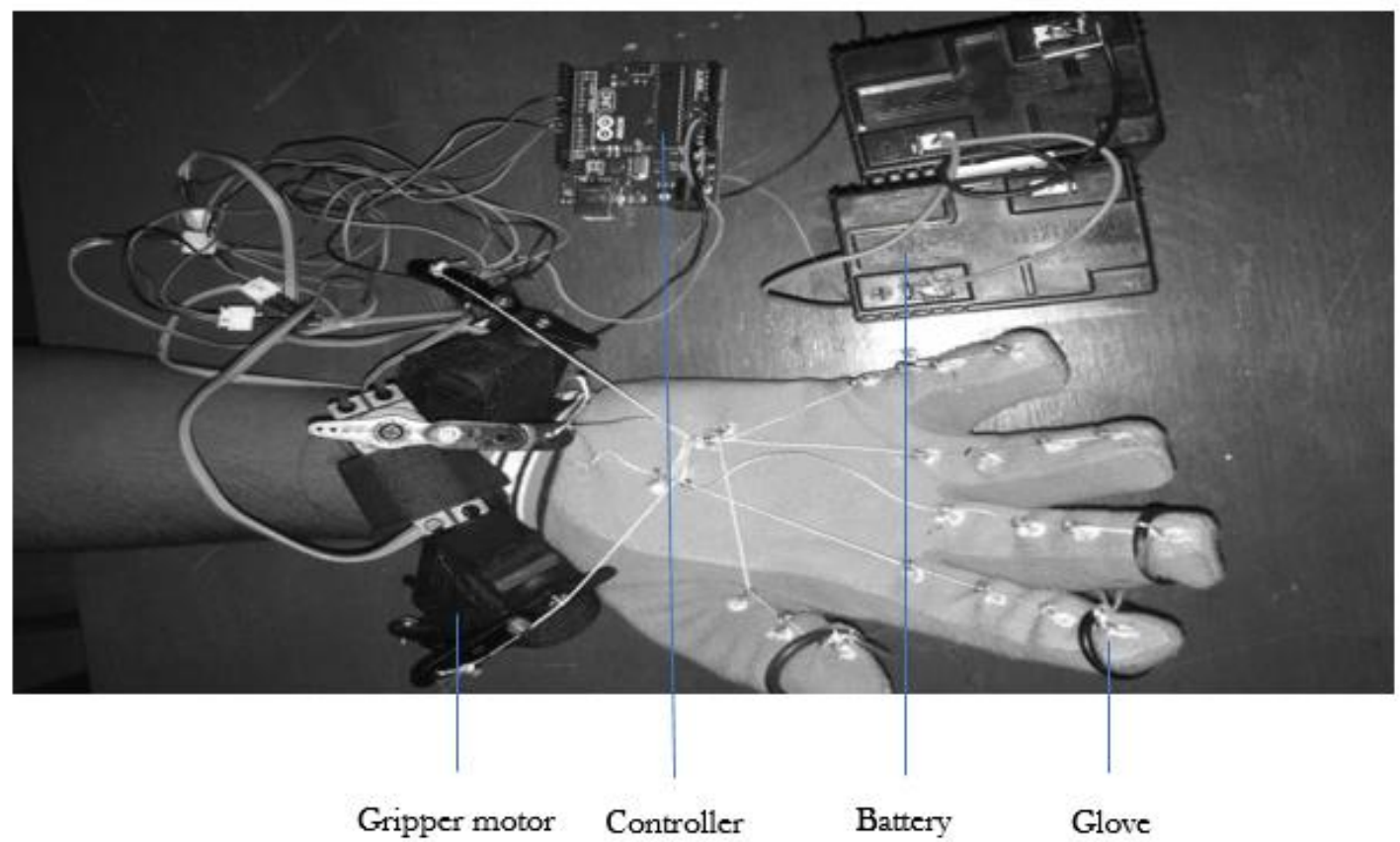

Figure 2 Experimental setup of Rehabilitation using Gripper Motor

\section{Result Analysis and Discussion}

A patient was reported with a known case of HTN (Hypertension). A periodic treatment was given over a period of 1 year to stabilize his blood pressure. On course of this treatment he was presented with complaint of numbness of left upper and lower limb. The patient was diagnosed with posterior circulation Cerebral- Vascular Accident (CVA) due to hypertension in the areas of Left Cerebellar bilateral occipital / Pons / midbrain / left thalamic infract. The patient was hospitalized and given adequate medical assistance for improving his health care status. Adequate physiotherapy measures were provided to him based on his condition during the course of hospital treatment. The patient developed some state of sense in his upper and lower limb and was able stand and walk with the support of two attenders, yet the tasks of holding and lifting objects for the accomplishment of daily living was a challenge for the patient. Hence the patient was assisted with the physiotherapy at the hospital. After a period of stay at the hospital the patient had developed some distress and was willing to move home. Considering his conditions, and after consultation from the physiotherapists the patient opted for home based rehabilitation therapy

\section{A. Fugl Meyer Assessment report using Gripper Motor Actuated Rehabilitation systems}

With reference to the assessment criteria set, the results displayed in table 2 were obtained from the patient after the experimental trial using the motor actuated rehabilitation system (gripper motor) with proper consent 
Vol. 3 Iss. 2 Year 2021

A. Kalaiarasi et al., /2021

Table 2. Fugl Meyer Assessment report for a patient using Motor Actuated Rehabilitation systems (Gripper motor)

\begin{tabular}{|l|c|c|c|c|c|c|}
\hline \multicolumn{1}{|c|}{ Parameter } & Null & Partial & Full & \multicolumn{3}{c|}{ Assessment } \\
\hline \multicolumn{1}{|c|}{ Assessment } & \multicolumn{3}{|c|}{ Scores } & Pre & Mid & Post \\
\hline Upper Extremity & 0 & 1 & 2 & 0 & 0 & 0 \\
\hline Flexors & 0 & 1 & 2 & 0 & 1 & 1 \\
\hline Extensors & 0 & 2 & 4 & 0 & 1 & 1 \\
\hline I.Reflex activity & 0 & 1 & 2 & 1 & 1 & 2 \\
\hline Shoulder retraction & 0 & 1 & 2 & 1 & 1 & 1 \\
\hline Shoulder elevation & 0 & 1 & 2 & 0 & 0 & 0 \\
\hline Shoulder abduction & 0 & 1 & 2 & 1 & 1 & 1 \\
\hline Shoulder external rotation & 0 & 1 & 2 & 0 & 0 & 0 \\
\hline Elbow flexion & 0 & 1 & 2 & 1 & 1 & 1 \\
\hline Forearm supination & 0 & 1 & 2 & 1 & 1 & 2 \\
\hline Shoulder adduction & 0 & 1 & 2 & 1 & 1 & 2 \\
\hline Elbow extension & 0 & 1 & 2 & 1 & 1 & 1 \\
\hline Forearm pronation & 0 & 1 & 2 & 0 & 0 & 0 \\
\hline II.Volatile motion within synergies & 0 & 9 & 18 & 7 & 7 & 10 \\
\hline Hand to lumbar spine & 0 & 1 & 2 & 0 & 0 & 0 \\
\hline Shoulder flexion & 0 & 3 & 6 & 1 & 1 & 1 \\
\hline Pronation-supination & 0 & 3 & 6 & 2 & 3 & 5 \\
\hline III.Volitional motion mixing synergies & 0 & 1 & 2 & 1 & 1 & 1 \\
\hline Shoulder abduction & 0 & 2 & 1 & 1 & 2 \\
\hline Shoulder flexion & 0 & 1 & 2 & 0 & 1 & 2 \\
\hline Pronation-supination & 0 & 1 & 1 & 1 \\
\hline IV.Volitional motion with no synergies & 0 & & & \\
\hline
\end{tabular}


Vol. 3 Iss. 2 Year 2021

A. Kalaiarasi et al., /2021

\begin{tabular}{|c|c|c|c|c|c|c|}
\hline V.Normal reflex activity & 0 & 1 & 2 & 1 & 1 & 2 \\
\hline TOTAL A (Upper Extremity) & 0 & 18 & 36 & 11 & 13 & 19 \\
\hline \multicolumn{7}{|l|}{ B. Wrist } \\
\hline Stability at $15 \%$ dorsiflexion & 0 & 1 & 2 & 0 & 1 & 1 \\
\hline Repeated dorsiflexion & 0 & 1 & 2 & 0 & 0 & 2 \\
\hline Stability at $15 \%$ dorsiflexion & 0 & 1 & 2 & 1 & 2 & 2 \\
\hline Repeated dorsiflexion & 0 & 1 & 2 & 1 & 1 & 2 \\
\hline Circumduction & 0 & 1 & 2 & 1 & 1 & 2 \\
\hline B.Wrist & 0 & 5 & 10 & 3 & 5 & 9 \\
\hline \multicolumn{7}{|l|}{ C. Hand } \\
\hline Mass flexion & 0 & 1 & 2 & 0 & 2 & 2 \\
\hline Mass extension & 0 & 1 & 2 & 1 & 1 & 2 \\
\hline Flexion in PIP and DIP & 0 & 1 & 2 & 0 & 2 & 1 \\
\hline Thumb adduction & 0 & 1 & 2 & 0 & 1 & 2 \\
\hline Opposition & 0 & 1 & 2 & 0 & 1 & 2 \\
\hline Cylinder grip & 0 & 1 & 2 & 1 & 1 & 1 \\
\hline Spherical grip & 0 & 1 & 2 & 1 & 1 & 1 \\
\hline C.Hand & 0 & 7 & 14 & 3 & 8 & 11 \\
\hline \multicolumn{7}{|l|}{ D. Coordination speed } \\
\hline Tremor & 0 & 1 & 2 & 1 & 1 & 1 \\
\hline Dysmetria & 0 & 1 & 2 & 1 & 1 & 1 \\
\hline Time & 0 & 1 & 2 & 1 & 1 & 1 \\
\hline D.Coordination speed & 0 & 3 & 6 & 3 & 3 & 3 \\
\hline Total A-D & 0 & 33 & 66 & 20 & 30 & 42 \\
\hline \multicolumn{7}{|l|}{ H. Sensation } \\
\hline Light touch upprarm,fore arm & 0 & 1 & 2 & 0 & 1 & 2 \\
\hline
\end{tabular}


Vol. 3 Iss. 2 Year 2021

A. Kalaiarasi et al., /2021

\begin{tabular}{|l|c|c|c|c|c|c|}
\hline Light touch palmer surface of hand & 0 & 1 & 2 & 0 & 1 & 1 \\
\hline Position Shoulder & 0 & 1 & 2 & 0 & 0 & 1 \\
\hline Position Elbow & 0 & 1 & 2 & 1 & 1 & 2 \\
\hline Position Wrist & 0 & 1 & 2 & 0 & 0 & 0 \\
\hline Position thumb & 0 & 1 & 2 & 0 & 1 & 2 \\
\hline H.Sensation & 0 & 6 & 12 & 1 & 4 & 8 \\
\hline I. Passive Joint Motion & & & & & & \\
\hline Shoulder & 0 & 4 & 8 & 3 & 3 & 4 \\
\hline Elbow & 0 & 2 & 4 & 2 & 3 & 4 \\
\hline Forearm & 0 & 2 & 4 & 1 & 2 & 3 \\
\hline Wrist & 0 & 2 & 4 & 2 & 3 & 3 \\
\hline Fingers & 0 & 2 & 4 & 2 & 2 & 2 \\
\hline I.Passive Joint Motion & 0 & 12 & 24 & 10 & 13 & 16 \\
\hline J. Joint Pain & & & & & & 3 \\
\hline Shoulder & 0 & 4 & 8 & 3 & 3 & 3 \\
\hline Elbow & 0 & 2 & 4 & 2 & 2 & 3 \\
\hline Forearm & 0 & 2 & 4 & 2 & 3 & 4 \\
\hline Wrist & 0 & 2 & 4 & 2 & 3 & 3 \\
\hline Fingers & 2 & 4 & 2 & 3 & 3 \\
\hline J. Joint Pain & 0 & 24 & 11 & 14 & 17 \\
\hline
\end{tabular}

\section{B. Graphical representation in terms of Scatter plot and Box plot}

Table 3 figures out the consolidated report of the results obtained from the table 2. The consolidated scores obtained during pre, mid and post phases of assessment were consolidated in the table.

The scatter plot shown in figure 3 infers how the major parameters of Fugl Meyer Assessment are affected over the period of assessment. The $\mathrm{x}$-axis predictor variable represents the number of weeks of assessment and the y-axis response variable represents the motion 
parameters of Fugl Meyer Assessment. The linear scatter plot obtained across the weeks demonstrates a linear model of data with no outliers.

Table 3 Consolidated Statement

\begin{tabular}{|l|l|l|l|}
\hline \multicolumn{1}{|c|}{ Parameter } & \multicolumn{1}{|c|}{ Pre-assessment } & \multicolumn{1}{c|}{ Mid-assessment } & \multicolumn{1}{c|}{ Post-assessment } \\
\hline A. Upper extremity & 12 & 16 & 19 \\
\hline B. Wrist & 3 & 5 & 9 \\
\hline C. Hand & 3 & 8 & 11 \\
\hline D. Coordination Speed & 3 & 3 & 4 \\
\hline Total A-D & 21 & 32 & 42 \\
\hline Sensation & 1 & 4 & 8 \\
\hline Joint pain & 10 & 13 & 16 \\
\hline Passive Joint Motion & 11 & 14 & 17 \\
\hline
\end{tabular}
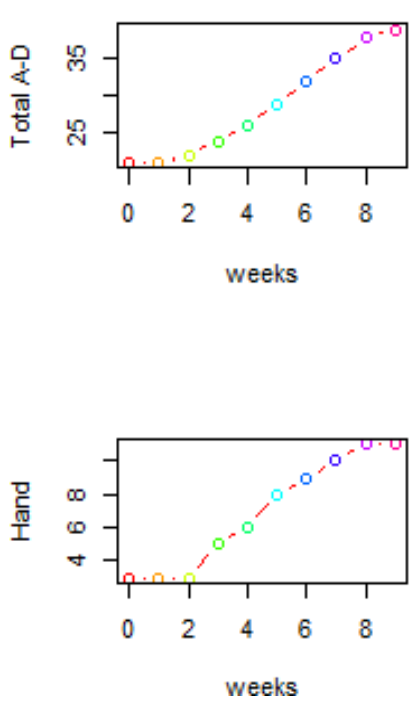
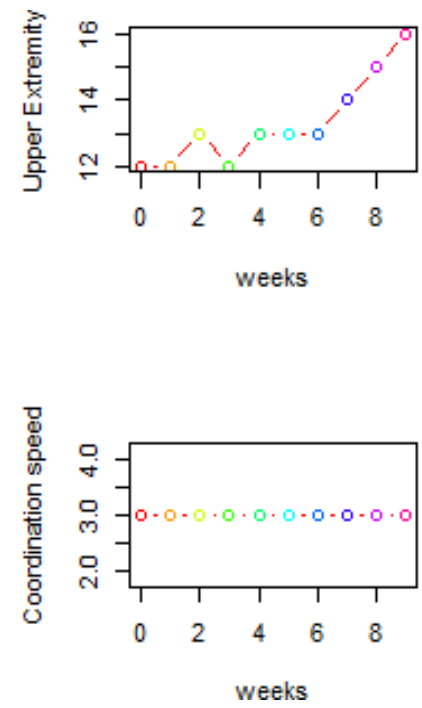
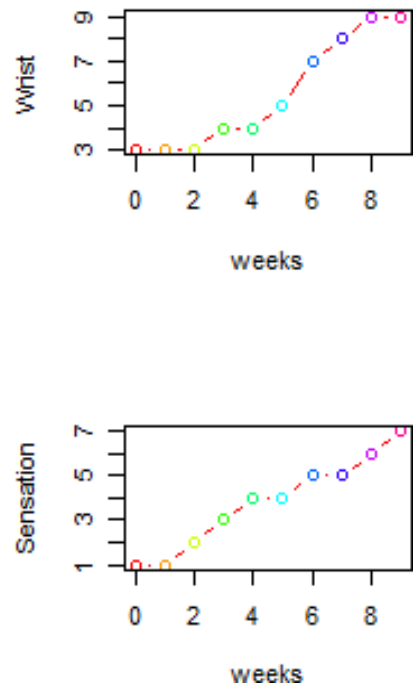

Figure 3 Scatter plot for Motor Actuated Rehabilitation system (Gripper motor)

The corresponding boxplot shown in figure 4 also proves the evidence for a normalized, clean data with no outlier extremes. The factors of Sensation, coordination speed, Hand, Upper Extremity, Wrist proves to vary normally across the weeks and hence the predictor and response variables prove to be in a linear modelling.Thus, the plots ensure reasonable linear improvements over the scale. 
Vol. 3 Iss. 2 Year 2021

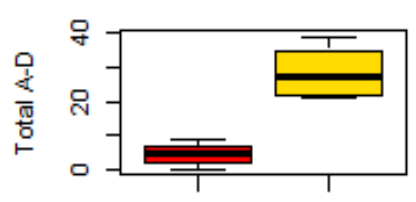

weeks

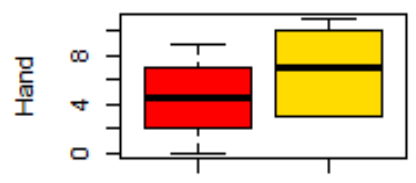

weeks

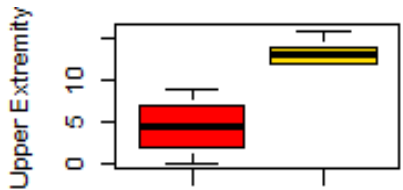

weeks

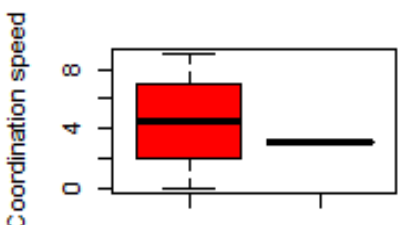

weeks

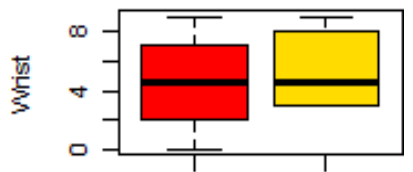

weeks

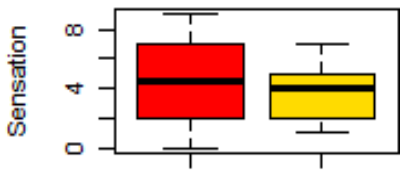

weeks

Figure 4 Box plot for Motor Actuated Rehabilitation system (Gripper motor)

\section{R-Tool Analysis of The Proposed Systems}

In order to deduce the computational complexity and human errors, the data obtained using Fugl Meyer Assessment is subjected to be analysed using R-tool. The data obtained from each patient is converted into an Electronic Health Record (EHR) and subjected to data cleansing. The cleansed data is converted into a Comma Seperated Value (.CSV) format. Each dataset is imported into the R-tool workspace using the command "read.csv". On execution of the code, the values of t,p, DoF (Degrees of Freedom), Mean Difference is extracted and it is listed in the table 4

Table 4: Statistical analysis results

\begin{tabular}{|c|c|c|c|c|c|c|}
\hline S. No. & Pre & Post & $t$ value & P value & DoF & Mean Diff \\
\hline 1 & 21 & 44 & 1.8 & 0.11 & 6 & 5.348 \\
\hline 2 & 22 & 43 & 1.8 & 0.11 & 6 & 5.751 \\
\hline 3 & 22 & 42 & 2.3 & 0.08 & 6 & 5.825 \\
\hline 4 & 18 & 36 & 1.8 & 0.14 & 6 & 4.715 \\
\hline 5 & 19 & 37 & 1.9 & 0.16 & 6 & 4.587 \\
\hline
\end{tabular}

From the table 3, it is observed that, the difference between the pre assessment status at week 0 and the post assessment status at week 10 shows a reasonable improvement in the Kinematics of motion of the patients subjected to trial. Also, the obtained t- values from all the five experimental trials seem to be positive. The p-values have confirmed that there exists a mean 

Vol. 3 Iss. 2 Year 2021
A. Kalaiarasi et al., /2021

difference between the two vectors „mee and „n $\mathrm{n}^{\mathrm{ee}}$ since they are greater than 0.05 . The mean difference is said to be positive from the observed positive, $\mathrm{t}^{\mathrm{e}}$ values.

Even in this case of the assessment performed by therapist, the datasets favor alternate hypothesis that signifies there is a change in the assessment made across the weeks. This positive change thus drives the functionality of the product in improvising the patients motion control .Thus according to the various assessments done with the help of $\mathrm{R}$ tool the stability of the product is recognized. Hence the product proves to be helpful in bringing about the movements of the upper arm of the patient thus bringing about noteworthy changes in the lifestyle of the stroke survival group. After the execution of the above mentioned models and implying on various patients on home restoration premise, different parameters were estimated and looked at. The correlations depended on development, outline, and executions are listed in Table 5 which briefs the examination of different parameters acquired from the results of the device.

Table 5 Result Analysis of the proposed systems for Upper Arm

Hemiplegia

\begin{tabular}{|c|c|c|}
\hline S. No. & Parameter & Result \\
\hline 1 & Energy Source & External \\
\hline 2 & Power Supply & Single phase \\
\hline 3 & Storage & Lead Acid battery \\
\hline 4 & Diversity & Good \\
\hline 5 & Hand Kinematics & Wrist \& Fingers \\
\hline 6 & Cost of a supply source & Low \\
\hline 7 & Actuators & Gripper motor \\
\hline 8 & Force & Medium \\
\hline 9 & Loss & Minimum \\
\hline 10 & Control & Yes \\
\hline 11 & Portability & Good \\
\hline 12 & Reliability & Good \\
\hline 13 & Size & Medium \\
\hline 14 & Weight & Less \\
\hline 15 & Large force capabilities & Medium \\
\hline 16 & Design Cost & Low \\
\hline 17 & Controller & Arduino \\
\hline
\end{tabular}




\section{Conclusion}

The outcome obtained from all the trial preliminaries adds that the rehabilitation device can be utilized for home based rehabilitation. The major advantages of this device are it is readily available, less time consumption and adequacy. The clinical professionals can also use the device for treatment purpose. On the other hand, this device can be extended to be used for Lower Arm Hemiplegia (LAH). The device can be developed with electromechanical frameworks which would help the patients to their lost motion skills.

\section{References}

[1] Ruidan Hu, Xiaoyan Wang, Zhihong Liu, Jiakun Hou, Yangyang Liu, Jinyi Tu, Miao Jia, Yue Liu \& Hongzhen Zhou, (2020). Stigma, depression, and post-traumatic growth among Chinese stroke survivors: A longitudinal study examining patterns and correlations, Journal of Topics in Stroke Rehabilitation, 29, 16- 29. https://doi.org/10.1080/10749357.2020.1864965

[2] Andreasen, D.S., Allen, S.K., \& Backus, (2015). Exoskeleton with EMG Based Active Assistance for Rehabilitation, 9th International Conference on Rehabilitation Robotics, 2005. ICORR 2005, IEEE, 333-336. https://doi.org/10.1109/ICORR.2005.1501113

[3] Jackson, P.L., Lafleur, M., Malouin, F., Richards, C., \& Doyon, J. (2001). Potential Role of Mental Practice Using Motor Imagery in Neurologic Rehabilitation, Archives of Physical Medicine and Rehabilitation, 82, 1133-1141. https://doi.org/10.1053/apmr.2001.24286

[4] Bansil Shalini, Prakash Neel, Kaye Joel, Wrigley Sandra, Manata Christina, StevensHaas Claire \& Kurlan Roger, (2012). Movement Disorders after Stroke in Adults: A Review, Tremor and Other Hyperkinetic Movements, 1-7. http://doi.org/10.5334/tohm.110

[5] Chen W., Hopfner F., Becktepe J.S., \& Deuschl G., (2017). Rest Tremor Revisited: Parkinson's Disease and Other Disorders, Translational Neurodegeneration, 6. https://doi.org/10.1186/s40035-017-0086-4

[6] World Health Organization., (2019). Preventing Chronic Diseases: A vital investment, World Health Organization, Geneva, Switzerland. https://apps.who.int/iris/handle/10665/43314

\section{Funding :}

No funding was received for conducting this study. 


\section{Conflict of interest :}

The Authors have no conflicts of interest to declare that they are relevant to the content of this article.

About The License : (C) The Author(s) 2021. The Text of this article is open access and licensed under a Creative Commons Attribution 4.0 International License 